\title{
Dynamics of multi-domain protein ER-60 revealed by small angle X-ray scattering data and molecular dynamics simulations
}

\author{
Masahiro Shimizu, Aya Okuda, Ken Morishima, Nobuhiro Sato, Rintaro Inoue, Reiko Urade, \\ Masaaki Sugiyama \\ Institute for Integrative Radiation and Nuclear Science, Kyoto University, Kumatori, Sennan-gun, Osaka, Japan \\ Shimizu.masahiro.3n@kyoto-u.ac.jp.
}

Small-angle X-ray scattering (SAXS) profile of a biomolecule reflects its meso- and nano- scale structure. Since the profile is contributed by all molecules in solution, the SAXS is a powerful method to study structural ensemble of the protein. We are establishing methods to elucidate structural ensemble of proteins at near-atomic resolution by combining SAXS and molecular dynamics simulations.

In this study we focused on structure and dynamics of multi-domain protein ER-60. ER-60 is a member of Protein disulfide isomerase family, which promote correct protein folding via isomerization of disulfide bonds. The ER-60 is composed four domains, a, b, b', and a'. Both a and a' domain have active Cys-Gly-His-Cys (CGHC) motif [1]. In each CGHC motif, two cysteines take either S-S (oxidized) or -SH (reduced) states. We have obtained SAXS profiles of ER-60 with both all CGHC oxidized (oxidized ER-60) and CGHC reduced (reduced ER-60). Our SAXS profiles did not match known crystal structure, and the SAXS profiles of the two states were slightly different from each other [2].

To investigate behaviour of ER-60 in solution, we performed multi-scale molecular dynamics simulations. First, fluctuation of each domain was examined by atomistic MD simulations. The fluctuation around active motif differed between oxidized and reduced ER-60, but no significant difference was seen in the other regions. It suggests that the difference of SAXS profile between two states is not due to the difference of intra-domain dynamics.

Second, motion of full-length ER-60 was examined by coarse-grained molecular dynamics (CGMD) simulations with CG Martini model [3], where each amino acid is represented by one to six particles. We have successfully obtained simulation trajectory which reproduce our SAXS profile. From the simulation trajectory, we analysed inter-domain interface and frequency of binding/dissociation of each pair of the four domains.

Third, structural difference between oxidized ER-60 and reduced ER-60 was studied by coarser CGMD simulations with AICG2+ model [4], which enable extensive structural-sampling. We compared simulation snapshots which reproduce SAXS profile of oxidized ER-60 with simulation snapshots which reproduce that of reduced ER-60. Our simulation showed that the difference of two SAXS profiles reflect the difference in position of a' domain [2].

[1] Kozlov, G., Määttänen, P., Tomas, D. Y. \& Gehring, K. (2010). FEBS J. 277, 3924.

[2] Okuda, A., Shimizu, M., Morishima, K., Inoue, R., Sato, N., Urade, R. \& Sugiyama, M. (2021). Sci. Rep. 11, 5655.

[3] Souza, P. C. T., Alessandri, R., Barnoud, J., Thallmair, S., Faustino, I., Grünewald, F., Patmanidis, I. et al. (2021). Nat. Methods 18, 382.

[4] Li, W., Wang, W. \& Takada, S. (2014). Proc. Natl. Acad. Sci. USA 111 (29), 11550.

Keywords: small angle X-ray scattering; multi-domain protein; ER-60; molecular dynamics simulation 